\title{
NEWER PREPARATIVE METHODS IN ORGANIC CHEMISTRY
} SYMPOSIUM was held jointly by the Chemical
Society and the Fine Chemicals Group of the
Society of Chemical Industry on "Newer Preparative
Methods in Organic Chemistry" at the Chemistry
Department, University College, London, on Novem-
ber 7 . The meeting took the form of two sessions in
the afternoon at each of which three papers were
read ; the reading of papers was followed by a
dinner in the evening, also at University College.
The afternoon meetings were attended by nearly 400
chemists and the evening dinner by 116. Papers
were read on "Dehydrogenation with Manganese
Dioxide" by Dr. R. M. Evans, "Amine Oxidations"
by Prof. H. B. Henbest, "Organic Oxidations with
Hexavalent Chromium" by Dr. R. Slack, "Mixed
Anhydrides as Synthetical Reagents" by Prof. G. W.
Kenner, "Some Reactions between Metal Carbonyls
and Acetylenic Compounds" by Dr. M. C. Whiting
and "Some Applications of Metal Carbonyls in
Organic Chemistry" by Dr. P. O. Lenel.

The meeting was opened by Prof. E. L. Hirst, who mentioned that this was the first joint meeting between the Chemical Society and the Fine Chemicals Group and expressed his hope that it would be a successful one. Dr. B. A. Hems took the chair at the first session and Prof. E. R. H. Jones presided over the second.

The purpose of the meeting was to discuss the applications of some reagents that have recently come into prominence in preparative chemistry and of which the scope and details of experimental methods may not yet be widely known. The authors of the papers had themselves had extensive personal experience of these reagents and could point to the theoretical and practical significance of various methods of using them.

Dehydrogenation by manganese dioxide was discussed by Dr. Evans. When this reagent is used for dehydrogenation the method of preparation and the physical form of the oxide appear to have a considerable effect on its reactivity. One form, prepared under strongly alkaline conditions, appears to be the best for dehydrogenating unsaturated primary or secondary alcohols to $\alpha: \beta$-unsaturated aldehydes or ketones, whereas an acidic form appears to be better for dehydroganating unsaturated ketones, at least in the sterol group. Dr. Evans pointed out that manganese dioxide brought about many other oxidizing reactions under more vigorous conditions, but that selectivity was then lost. The most useful applications of this reagent lay with specially active forms of the oxide used in mild conditions on polyene or polyenyne alcohols that were difficult to oxidize to the corresponding aldehydes or ketones by other methods.

The dehydrogenation of amines to give unsaturated compounds was discovered many years ago but has received very little application in general preparative chemistry. It is to be expected that the work that Prof. Henbest and his colleagues are doing on the subject will bring this potentially useful reaction to the notice of a wider audience.

He mentioned in his paper that tertiary amines are generally regarded as being chemically rather unreactive, but pointed out that this is not so. Dehydrogenation with mercuric acetate under mild conditions in dilute acetic acid gives enamines. Sometimes in a heterocyclic ring system complete dehydrogenation to the aromatic ring will take place, but often partial dehydrogenation can also occur. $\mathrm{He}_{\theta}$ compared this with the action of manganese dioxide on tertiary amines, where again enamines are formed under mild conditions, but this is often accompanied or followed by oxidative cleavage of the double bond to give amides. Diethylaniline gives a mixture of ethylvinyl aniline and ethylformanilide.

The third of the oxidizing agents discussed, chromic oxide, is, of course, widely used for a variety of purposes. It may be applied to both dehydrogenations and oxidations by a diversity of methods the theoretical and practical significance of which is not yet thoroughly understood. Differences undoubtedly exist about the effectiveness with which these different techniques can be used on different substrates, and although it is probable that a common theoretical background exists, it is nevertheless true that much has yet to be learnt about the best method to be used on any particular type of substance. Dr. Slack pointed out that oxidations with chromic oxide in acetic acid were often not stoichiometric, that chromic oxide could still remain, even in the presence of unoxidized substrate, because its oxidizing action was inhibited by the presence of lower valency forms of chromium. He added that one function of the sulphuric acid, sometimes added to these reactions, is to overcome this inhibition. He compared the uses of chromic oxide as its pyridine complex or tertiary butyl ester and finally pointed to the existence of some other hexa- and penta-valent chromium complexes such as acetyl chromic acids or complex salts derived from trichloro oxo-chromium (V) that had not yet been investigated by organic chemists but might repay some attention.

These three papers composed the first session of the meeting and were followed by three on condensation reagents.

Mixed anhydrides have come very much into prominence during the past few years for condensations involving carboxyl groups where, because of the delicacy of either the starting materials or the final product, mild reaction conditions are required.

Another advantage that mixed anhydrides have is that one of the partners is usually derived from a small and relatively cheap molecule, and their use avoids the wasteful regeneration of a molecule of the larger and perhaps much less accessible acid. Prof. Kenner pointed out that in a mixed anhydride a cleavage reaction could theoretically take either of two directions and he discussed the relative activities of various groups showing that the attack of a nucleophilic reagent was determined by the relative electrophilic character of the two parts of the anhydride. This, however, was not the only factor affecting reaction; the $p K$ of the acids taking part, steric hindrance and the co-ordination number at the electrophilic centres also played a part in determining which of the two reaction products would predominate. Mixed anhydrides derived from chloroformic ester, phosphoric and sulphuric acids or their esters had shown themselves to be extremely useful in the synthesis of poptides and nucleosides. 
The last two papers were on metal carbonyls. Frequently to-day, new preparative methods involve the use of reagents that a fow years ago were considered too difficult or too dangerous to handle because of the risks of explosion, fire or toxicity. The hazards of using acetylene under pressure and the toxicity of carbonyls frequently cause these reagents to be avoided and it is hoped that these pepers, in which safe methods of handling were discussed by the authors, may alleviate some of these fears.

Dr. Whiting stressed the usefulness of nickel carbonyl in reacting with acetylenes having a terminal triple bond to give $\alpha$-substituted acrylic acids, a type of compound otherwise rather inaccessible. These resctions are carried out in acetic acid at moderate temperature, with excess carbonyl which is not difficult to handle provided that its transfer from vessel to vessel is carried out under conditions involving only a very slight degree of exposure to air, and provided that excess is removed from the reaction mixture by co-distillation with ether. The distillate is immediately disposed of and leaves a reaction mixture that can safely be handled in the usual way. Even so, both he and Dr. Lenel pointed out that although these substances could be handled safely they still had to be treated with considerable respect. Dr. Whiting also went at some length into the mechanisms of these reactions. He was followed by Dr. Lenel, who dealt mostly with the industrial applications of carbonyls in reaction with acetylenes to give unsaturated acids, or with olefines to give saturated acids. Cobalt carbonyl catalyses the important reaction of carbon monoxide and hydrogen with olefines to give aldehydes which can then be reduced to alcohols by hydrogenation. The industrial preparation of alcohols and acids by these reactions is now carried out on a very large scale involving many thousands of tons of product per annum, though the reactions necessitate fairly high temperatures and pressures. Several other carbonyls of transitional elements are known, though they have not yet achieved prominence in an industrial sense; they are of profound interest to the inorganic and theoretical chemist because their constitutions throw new light on electronic structures.

This was, as Prof. Hirst pointed out, the first joint meeting of the Chemical Society and the Fine Chemicals Group of the Society of Chemical Industry, and it is very much to be hoped that it will be followed by others. The members of the Fine Chemicals Group are predominantly interested in the pre. paration of complex chemicals in a high degree of purity : perfumes, photographic sensitizers, drugs, vitamins and hormones, for example. These processes often involve twelve to twenty stages that, if they are to be carried out efficiently, demand the use of the most up-to-date knowledge and techniques available. This Group, of all the special groups in the Society of Chemical Industry, is the one that has the strongest kinship with the Chemical Society. With the rapidly increasing activity in industrial chemistry of all kinds and the rapid extension of industrial technical staffs, joint meetings of this kind can be a valuable aid to progress. A closer relationship between the members of the two Societies can be of great benefit to both.

\section{INTERACTION IN IONIC SOLUTIONS}

$\mathrm{T}$ HE Faraday Society held a general discussion on "Interaction in Ionic Solutions" at Oxford during September 17-19. The meeting was held in the University Laboratory of Physiology, and more than 180 members and visitors stayed in Balliol College, where the guest-night dinner was held. The president of the Society, Mr. R. P. Bell, in opening the discussion, reminded those present that a general discussion on "Electrolyte Solutions" had been held thirty years ago in Oxford. In fact, the president's first published contribution to the subject was presented at that meeting in 1927 in a joint paper with Sir Harold Hartley. Among others present, who had attended and contributed to the earlier discussion, was Prof. H. S. Harned (Yale), who delivered the Spiers Memorial Lecture on "Some Recent Experimental Studies of Diffusion in Liquid Systems". Prof. Harned gave an account of the elegant experimental techniques for the precision measurement of diffusion in electrolytes which have been recently developed at Yale, and indicated some of the many possible directions of further advance. The accurate determination of activity coefficients by diffusion measurements is a remarkable achievement.

The first paper to be discussed was that by Prof. H. Falkenhagen and Dr. G. Kelbg (Rostock), which dealt with the extension of the limiting-law equations. Dr. M. Eigen (Göttingen) directed attention to the information that can be obtained about the intimate details of short-range interaction in bi-bivalent electrolytes by the observations of relaxation effects in sound-absorption spectra. Dr. S. Levine and Mr. H. E.
Wrigley (Manchester) reported a calculation of the interaction of two univalent ions at small separations, and considered the relevance of the macroscopic dielectric constant of the solvent in this situation. In discussion, Prof. L. Onsager (Yale) directed attention to some recent mathematical advances which might help in the electrolyte problem, while Prof. H. S. Frank (Pittsburgh) seized the opportunity to express heretical views about the Debye-Hückel theory. A paper by Prof. T. F. Young, Mr. Y. C. Wu and Dr. A. A. Krawetz (Chicago) reported some measurements of the heat effects due to interactions between ions of like charge when pairs of univalent electrolytes with an anion or cation in common are mixed.

There followed a major section devoted to the presentation and discussion of papers dealing with the incomplete-dissociation model as a way of handling short-range ion-ion interactions. Prof. C. W. Davies (Aberystwyth) surveyed the present position in an introductory paper. Prof. E. A. Guggenheim (Reading) considered the ion distribution in dilute aquoous solutions of single binary electrolytes, and concluded that although the Debye-Hückel approximation is invalid for bi-bivalent sulphates in water, the model (complete ionization to rigid spherical ions) is satisfactory if, following $N$. Bjerrum, ions of opposite charge approaching more closely than 10-14 A. are treated as associated. Dr. O. Redlich and Dr. G. C. Hood (Shell Development Co., California) presented the results of Reman and nuclear magnetic-resonance investigations of the dissociation of strong acids (including nitric, perchloric and sul- 